\title{
APRENDIZAGEM DA AVALIAÇÃO DA VITALIDADE DO RECÉM-NASCI- DO PELO MÉTODO DE APGAR
}

Elsbeth Fïrstenau Schlatter *

SCHLATTER, E. F. Aprendizagem da avaliação da vitalidade do recém-nascido pelo método de Apgar. Rev. Esc. Enf. USP, São Paulo, 15(3):267.273, 1981.

Este trabalho apresenta um relato de estudo sobre o ensino prático da avaliação do recém-nascido pelo método de Apgar, realizado com 16 alunas de habilitação em enfermagem obstétrica que avaliaram 43 recém-nascidos.

\section{INTRODUÇÃO}

A avaliação do estado geral do recém-nascido ( $R N)$ nos primeiros minutos de vida é de fundamental importância pois ela orienta a assistência imediata que o RN deverá receber e alerta quanto a problemas que poderão surgir. De todos os métodos de avaliação da vitalidade do $\mathrm{RN}$, o mais aceito e utilizado pelos profissionais que prestam assistência ao $\mathrm{RN}$, no mundo ocidental, é o método de Apgar.

APGAR (1953) publicou um esquema que estabelece a observação de cinco sinais em RN que indicam as condiçōes dos aparelhos respiratórios, cardiovascular e neuromuscular, a saber: freqüência dos batimentos cardíacos, tipo de freqüência dos movimentos respiratórios, tonus muscular, presença de reflexo mediante um estímulo e cor da pele. Este sinais são avaliados aos 60 segundos de vida e a cada sinal atribui-se uma nota variável de zero a dois, de acordo com as condições do RN. A avaliação da vitalidade é obtida somando-se os pontos atribuidos a cada sinal, sendo de dez o valor máximo. A nota dez corresponde a um RN em perfeitas condições e a nota zero, a um RN morto. O RN com Apgar de 7 a 10 é considerado em boas condições; com Apgar de 4 a 7 é considerado moderadamente deprimido e com Apgar de 0 a 3, gravemente deprimido.

Descrição dos sinais de Apgar segue:

Classicamente os cinco sinais do esquema de Apgar são pesquisados como

Freqüência cardíaca - Freqüência cardíaca entre 160 a 170 ou abaixo de 100 batimentos por minuto é considerada anormal. Segundo os autores, a freqüência cardíaca deve ser auscultada com estetoscópio ou por palpação do cordão umbilical, na junção do cordão com a parede abdominal. A ausência de batimentos indica pressão sanguinea baixa e débito cardíaco pobre. Em condições nor-

- Professor Assistente do Departamento de Enfermagem Materno-Infantil e Psiquiátrica da EEUSP, disciplina Enfermagem Obstétrica e Neonatal. Mestre em Enfermagem. 
mais, o cordão para de pulsar entre 10 e 30 segundos após o nascimento. A frequiência cardíaca é o sinal mais importante; quando o RN é oxigenado e há um aumento da mesma, isto constitui um sinal de melhora no estado geral. Freqüência acima de 100 , recebe a nota 2 ; de 1 a 100 , nota $I$; nota zero é atribuída quando não houver batimentos cardíacos.

Esforço respiratório - Este sinal é o segundo na ordem de importância. Se o esforço respiratório for vigoroso, o $\mathrm{RN}$ recebe nota 2; caso esteja presente, mas irregular e ineficaz, recebe nota 1; caso haja apnéia, é dada nota zero. Quanto mais irregular a respiração tanto mais acentuada a acidose respiratória e metabólica.

- Tonus muscular - Quando há flexão dos membros superiores e inferiores que resistem à extensão, e na presença de movimentação subseqüente adequada de todas as extremidades, o RN recebe nota 2 ; tonus "regular" recebe nota 1 , e, em caso de flacidez total, é lhe atribuida nota zero.

Irritabilidade reflexa - Este item pode ser testado de diversos modos. 0 objetivo da pesquisa da mesma é verificar a resposta a qualquer forma de estímulo. Este procedimento pode ser levado a efeito por meio de duas a três palmadas na planta do pé do $\mathrm{RN}$ ou introduzindo-se um cateter de borracha no nariz ou no orofaringe. Caso a resposta seja choro, o RN recebe nota 2; caso faça careta, mas não chore, recebe nota $l$; se não houver reação alguma, atribui-se a nota 0.

Cor - $\mathrm{O}$ item cor é o menos importante e o mais óbvio. Se a pele do recémnascido estiver toda rosada, recebe nota 2 , o que pode ocorrer em $15 \%$ dos recém-nascidos; a maioria dos RN tem a pele rosada, com exceção dos pés e das mãos, que se apresentam cianóticas, caso em que recebem nota 1. Quando todo o corno estiver cianótico ou pálido, recebe nota zero.

\section{Revisão bibliográfica}

BERRIEN (1961), EWERBECK (1967), LISBOA (1968), DELASCIO \& GUARIENTO (1968), FINSTER (1970), COCKBURN (1971), MAYRHOFER (1971), GREGORY (1972) GUPTA \& MOORE (1972) ressaltam genericamente a importância da avaliação do RN e do treinamento da equipe de saúde para realizar eficientemente a avaliação do mesmo. Outros autores são mais específicos, como AULD (1974) que descreve sobre a reanimação do RN, afirmando que o Apgar fornece uma estimativa das condições do neonato e critérios eficientes quando não houver tempo disponível para medir os gases sanguíneos. Estabelece, ainda, correlação grosseira entre o Apgar do primeiro minuto e o $\mathrm{pH}$ sanguíneo, como por exemplo: Apgar 1-3, pH 6,9 a 7,0; Apgar 4-7 e pH 7,0 a 7,20; Apgar 8-10 e pH 7,25. O mesmo autor ressalta ainda as medidas de reanimação dos RN, de acordo com o Apgar encontrado.

BOENISCH \& SALING (1974), referindo-se ao esquema Apgar, afirmam que este tem a desvantagem de não fornecer valores exatos, mensuráveis, e recomendam que, juntamente com o mesmo, seja avaliado o $\mathrm{pH}$ sanguíneo do RN.

ISSEL et alii (1976), reportando-se igualmente ao esquema Apgar e à sua determinação prognóstica para o periodo neonatal, concluem, após estudo reali- 
zado com 6778 recém-nascidos, que as diferenças de avaliąão, segundo este esquema, dependem do peso do $\mathrm{RN}$ ao nascer. Os mesmos autores afirmam que os dados patológicos em $\mathrm{RN}$, com peso igual ou inferior a $2500 \mathrm{~g}$, são três a quatro vezes mais freqüentes que em $R N$ com peso normal $(2501 \mathrm{~g}$ a $4500 \mathrm{~g})$. A existência de estreita relação entre o índice de Apgar e o peso do RN ao nascer foi também observada por MARAZZINI \& SANTUCCI (1977).

Alguns autores, como SERUNIAM \& BROMANN (1975), estabelecem correlação entre os esquemas de Apgar e o de Bayley, esquema este que avalia o desenvolvimento das funções psicomotoras de crianças com poucos meses de vida. Realizaram um estudo com crianças no oitavo mês de vida, submetendo-as a testes psicológicos do esquema de Bayley, e, de acordo com o resultado, cada uma delas foi classificada do seguinte modo: normal, suspeito e anormal. Segundo os autores, os resultados mostram correlação significativa entre o esquema de Apgar e o de Bayley, no oitavo mês de vida, pois a classificação anomal segundo este último esquema, é muito mais comum em crianças que apresentavam Apgar baixo ao nascer, quando comparadas com crianças classificadas como suspeito ou normal que apresentavam Apgar mais elevado.

Estabelecendo uma relação entre o esquema de Goodwin (alto risco materno) e o de Apgar, YEH et alii (1977) afirmam que esta relação não é surpreendente, pois, as condições da gestação tanto quanto os eventos do trabalho de parto, influem sobre a vitalidade do RN.

MARX et alii (1977) reportando-se à correlação entre dados bioquímicos e o índice de Apgar do primeiro minuto de vida, afirmam que as medidas de reanimação do $\mathrm{RN}$, no primeiro minuto, poderão alterar marcantemente as condições destes, após 60 segundos de vida e, por isso, pleiteiam a avaliação imediata após o nascimento.

Ao identificar as causas subjacentes à alta mortalidade neonatal, NAYE (1979) afirma que as infecções do líquido amniótico são responsáveis por uma grande porcentagem de mortes neonatais, associadas a índices baixos do esquema Apgar.

Alguns autores se preocupam com a execução prática do esquema Apgar e com o preparo do pessoal que executa esta atividade.

LISBOA (1968) ressalta em seu trabalho que o início da contagem dos pontos na avaliação da vitalidade do $\mathrm{RN}$, pelo método de Apgar, constitui um passo importante. A contagem, segundo este autor, "começa quando a cabeça e o pé do RN forem visíveis". A primeira avaliação será indicada, de preferência, após 55 segundos de vida. Alega o autor que muitos responsáveis pela avaliação calculam mais ou menos a partir do primeiro minuto, o que poderá dar margem a erro; sugere a colocação de um relógio de tempo, como os de laboratório, na sala de parto, com sinais de alarme auditivo, para que acuse os 55 segundos de vida do RN. Ressalta, também, que não há necessidade de esperar um minuto para iniciar a reanimação em crianças que nascem com anóxia grave. Nestes casos, a reanimação deve ser iniciada imediatamente após o parto e a avaliação deve ser realizada após a instalação da ventilação pulmonar.

FINSTER (1970), no seu trabalho sobre ressuscitação do RN, declara que, apesar dos progressos da obstetrícia e da anestesiologia, ainda nasce um número 
considerável de $\mathrm{RN}$ com problemas respiratórios que recebem nota igual ou inferior a 6, no primeiro minuto de vida; atribui este Apgar baixo às drogas usadas durante o trabalho de parto ou durante o parto, aos traumas de trabalho de parto precipitado, à obstetrícia operatória e à asfixia do RN durante o nascimento. Salienta o mesmo autor, a importância do treinamento de todos os membros da cquipe da sala de parto, para a ressuscitação, tanto do RN como da mãe.

COCKBURN (1971) relata que em $45 \%$ dos hospitais de ensino na Escócia, há pediatras responsáveis pela reanimação do RN e que, em mais de $80 \%$ de todos os hospitais do Reino Unido, onde não há pediatras disponíveis, o anestesista executa a ressuscitação do RN. Refere-se também ao esquema de Apgar, empregado no primeiro minuto após o nascimento e repetido no quinto minuto de vida. O mesmo autor alega, ainda, que o Apgar do primeiro minuto dá a indicação da necessidade de reanimação ativa, relacionada à acidose, e salienta que o Apgar do quinto minuto mostra maior correlação com os danos cerebrais subseqüentes.

MAYRHOFER (1971), analisando a situação no hospital da Universidade de Viena, descreve que o esquema de Apgar é realizado aos 60 segundos ou aos 90 segundos, após o nascimento. Alega também que os principais sinais para a avaliação do RN são o tonus muscular, os movimentos espontâneos e a respiração. Recomenda também que cada médico obstetra esteja apto para realizar a respiração artificial e a intubação endotraqueal.

ZIEGEL \& CRANLEY (1980) afirmam que a avaliação do RN pelo esquema de Apgar deve ser feita por uma das pessoas da sala de parto, inclusive a enfermeira, pois, esta avaliação não interfere com outros cuidados prestados ao RN. O tempo establelecido para a primeira avaliação é de 55 a 60 segundos após o nascimento. Recomendam, ainda, a determinação precisa da hora do nascimento. Não estabelecem uma ordem de seqüência para cada item do esquema, devendo caber isto à pessoa que faz a classificação e de acordo com a situação do RN, por exemplo: se o RN chorou imediatamente após o nascimento, o observador poderá querer verificar primeiramente a respiração e a cor, por outro lado, quando houver ausência de movimentos respiratórios e depressão do estado geral do $\mathrm{RN}$, o mesmo poderá pesquisar primeiramente o tonus muscular, a irritabilidade reflexa e os batimentos cardíacos.

\section{AVALIAÇÃO DO INDICE DE APGAR PELOS ALUNOS}

Em nosso meio, nas salas de primeiros cuidados ao $\mathrm{RN}$, nos centros obstétricos de hospitais de ensino, é comum encontrar-se, afixado à parede, um quadro contendo o esquema de Apgar. As palavras e os números contidos neste quadro, à primeira vista, parecem simples e claras, fáceis de serem entendidos. 0 mesmo acontece com o esquema de Apgar transcrito na ficha do RN. É raro ouvir por parte dos alunos de enfermagem alguma dúvida quanto à interpretação e aplicação do quadro. Entretanto, no momento em que o aluno tenta aplicar este esquema na prática, surgem algumas perguntas, tais como: Quando inicio a contagem? Em que seqüência avalio o RN? Qual a maneira mais fácil e rápida de avaliar cada item? Como conjugar a reanimação do $\mathrm{RN}$ com a avaliação?

$\mathrm{Na}$ revisão da literatura não encontramos trabalhos sobre o ensino prático do método de Apgar. 
Tendo em vista a importância da avaliação da vitalidade do RN nos primeiros minutos de vida e a necessidade de realizar essa avaliação com eficiência e com o mínimo de tempo possível, propusemo-nos a fazer este trabalho com o objetivo de padronizar o procedimento do método de Apgar e determinar o número de avaliaçoos necessárias para que, em trinta seguindos, o aluno seja capaz de avaliar corretamente a vitalidade do RN.

Os dados deste estudo foram colhidos durante o período de estágio no centro obstétrico, por 16 estudantes de habilitação em enfermagem obstétrica que avaliaram a vitalidade de $43 \mathrm{RN}$ de termo, de partos por via vaginal (normal e forceps), pesando de 2500 e $3800 \mathrm{~g}$. Todas as alunas receberam o mesmo ensino teórico-prático sobre a avaliação da vitalidade do recém-nascido, pelo esquema de Apgar, antes de iniciar a primeira avaliação.

A avaliação da vitalidade do RN, pelo esquema de Apgar, foi feita pela estudante orientada pela professora, que permaneceu durante toda avaliação a seu lado; utilizamos um cronômetro para determinar a hora exata do nascimento e o primeiro e o quinto minutos de vida, após expulsão total do concepto. Iniciamos a avaliação pela contagem da freqüência cardíaca, com o auxílio do estetoscópio clínico, colocado sobre a região pré-cordial; simultaneamente avaliamos a cor e a respiração do RN. Em seguida pesquisamos a irritabilidade reflexa, aplicando uma palmada tangencial na planta do pé do RN. Finalmente, testamos o tonus muscular, tentando extender os membros superiores e inferiores, delicadamente, a fim de verificarmos a resistência dos mesmos e dando a nota de acordo com a extensão dos membros e a resistência a esta extensão. Somamos todas as notas, sendo o total o Apgar do RN. No quinto minuto de vida, utilizando o mesmo procedimento foi realizada a segunda avaliação do RN pela mesma aluna.

Os dados obtidos não são suficientes para um tratamento estatístico que nos permita chegar a conclusões gerais, porém, podemos evidenciar algumas observações importantes tanto para a padronização na aplicação do método de Apgar, como para o ensino da mesma. Em relação à contagem da freqüência cardíaca fetal, não conseguimos realizá-la palpando o cordão umbilical, próximo à junção com a parede abdominal, devido a várias circunstâncias que impediram o livre acesso ao RN: campos esterilizados, conduta obstétrica em relação à seç̧ão do cordão umbilical, antes de completar o primeiro minuto de vida do RN ou parada dos batimentos cardíacos antes do primeiro minuto de vida. A contagem deste item não ofereceu dificuldade para a aluna, pois, na maioria dos casos, esta conseguiu identificar rapidamente a mesma.

Para dar as notas correspondentes à cor e à respiração, a aluna levava mais tempo que no item anterior, não lembrando de imediato os pormenores de cada um.

$\mathrm{Na}$ avaliação do tonus muscular, as alunas encontraram certa dificuldade quando se tratava de casos intermediários, como por exemplo, flexão incompleta das extremidades. Onde esta inicia e onde termina? Achamos que a aplicação da nota 1 , para este item, merece maior precisão. Na avaliação do quinto minuto, notamos que a aluna geralmente conseguia lembrar mais detalhadamente todos os itens da avaliação, sem precisar ser lembrada pela professora, embora o tempo utilizado não apresentasse diferença entre uma e outra avaliação, principalmente nas três primeiras experiências. 
Em relação ao tempo estipulado para a avaliação do Apgar e o número de experiências por estudante, conseguimos observar o seguinte: sete $(44 \%)$ alunas tiveram apenas uma única oportunidade de aplicar o esquema Apgar, que corresponde a duas avaliações, ou seja, a do primeiro e a do quinto minuto de vida do $\mathrm{RN}$; três $(19 \%)$ alunas tiveram duas; uma $(6 \%)$ teve quatro experiências; quatro $(25 \%)$ tiveram cinco e uma $(6 \%)$ teve seis experiências. Vários fatores são responsáveis por estas diferenças: rodízio dos alunos, horário de estágio, número de partos ocorridos no periodo. Devido ao rodízio dos alunos em diferentes locais de estágio, não foi possível aos mesmos repetirem a experiência, pois não voltavam mais ao centro obstétrico. Por outro lado, aconteceu várias vezes que ocorriam partos simultaneamente, sendo possível a cada aluno atender apenas a um RN, no que se refere à avaliação do Apgar. O tempo utilizado pelas alunas para avaliação do $\mathrm{RN}$, em média, foi o seguinte:

- Primeiro grupo, sete alunas (única experiência):

Apgar do primeiro minuto: $2 \min 3 \mathrm{~s}$;

Apgar do quinto minuto: $1 \min 2 \mathrm{~s}$;

- Segundo grupo, três alunas:

Primeira experiência:

Apgar do primeiro minuto: $1 \mathrm{~min}$

Apgar do quinto minuto: $1 \mathrm{~min} 13 \mathrm{~s}$;

Segunda experiência:

Apgar do primeiro minuto: 1 min $13 \mathrm{~s}$;

Apgar do quinto minuto: $1 \min 6 \mathrm{~s}$;

- Terceiro grupo seis alunas;

Primeira experiência:

Apgar do primeiro minuto: $1 \mathrm{~min} 18,6 \mathrm{~s}$;

Apgar do quinto minuto: 1 min $12,6 \mathrm{~s}$;

Segunda experiência:

Apgar do primeiro minuto: $1 \mathrm{~min} 2,4 \mathrm{~s}$;

Apgar do quinto minuto: 1 min $8 \mathrm{~s}$;

Terceira experiência:

Apgar do primeiro minuto: $1 \min 10 \mathrm{~s}$;

Apgar do quinto minuto: $53,8 \mathrm{~s}$;

Quarta experiência:

Apgar do primeiro minuto: $48,6 \mathrm{~s}$;

Apgar do quinto minuto: $50,8 \mathrm{~s}$;

Quinta experiência:

Apgar do primeiro minuto: $57 \mathrm{~s}$;

Apgar do quinto minuto: $49,6 \mathrm{~s}$;

Sexta experiência:

Apgar do primeiro minuto: $40 \mathrm{~s}$;

Apgar do quinto minuto: $30 \mathrm{~s}$;

\section{COMENTÁRIOS}

A partir deste estudo de ensino prático achamos oportuno destacar alguns aspectos, como segue: 
- Padronização do método de Apgar por meio de:

- Determinação precisa da hora do nascimento pelo uso do cronômetro;

- Início da avaliação, segundo Apgar, no primeiro minuto de vida;

- Contagem da freqüência cardíaca com estetoscópio clínico;

- Observação em ordem de seqüência, da respiração, da cor e do tonus muscular;

- Pesquisa da irritabilidade reflexa por meio do cateter de aspiração de secreções do orofaringe do $\mathrm{RN}$, porque a mencionada aspiração é uma medida comum entre os primeiros cuidados ao $\mathrm{RN}$, na maioria das maternidades;

- Avaliação do Apgar do quinto minuto de vida, na mesma seqüência citada.

Número de experiências por estudante:

- Foram necessárias, no mínimo, três experiências, para as alunas conseguirem fazer a avaliação em 30 segundos.

SCHLATTER, E. F. Learning the evaluation of the newborn's vitality by Apgar score. Rev. Esc. Enf. USP, São Paulo, 15(3):267-273, 1981.

The author describes the learning of the evaluation of the vitality of 43 newborn infants according to Apgar score by 16 nurse-midwifery students in a Sâo Paulo Maternity Hospital.

\section{REFERENCIAS BIBLUOGRAFICAS}

APGAR, V. A proposal for a new method of evaluation of the newborn infant. Curr. Res. Anesth. Analg, Rocky River, 32 (6): 260-7, Nov-Dec, 1953.

AULD, P. Resuscitation of the newborn. Amer. J. Nurs., New York, 74: 68-70, Jan. 1974.

BERRIEN, C. D. Evaluating the newborn. Nurs. Outlook, New York, 9 (2): 93-5, Feb. 1961.

BOENISCH, H. \& SALING, E. A combined clinical-biochemical scoring of the newborn. J. Perinat Med. New York, 2 (2): 122-9, 1974.

COCKBURN, F. Resuscitation of the newborn. Brit J. Anaesth. Cheshire, 43: 886-902, Sept., 1971.

DELASCIO, D. \& GUARIENTO, A. Anóxia. In: EMERGenCias no período neonatal. São Paulo, Centro de Estudos Perinatais de São Paulo, 1968.

EWERBECK, H. Zur Prophylaxe von Zerebralschäden beim Neugeborenen. Deut Med Wochenzeitsch, Stuttgart, 34: 1485-8, 25 Aug 1967.

FINSTER, M. Resuscitation of the newborn. Acta anaest Scandin, Aarhus, 37 (suppl): 86-93, 1970.

GUPTA, G. \& MOORE, J. Clinical trial of Doxopran Hydrochlories in the resuscitation of the newborn. Brit. J. Anaesth, Cheshire, 44: 626, June 1, 1972.

GREGORY, G. Respiratory care of newborn infants. Pedlat Clin N Amer. Philadelphia, 19: 311-24, May 1972.

ISSEL, F. P. et alii. Der Apgar-Wert des Neugeborenen and seine prognostische Aussagekraft fur den Verlaui de Neonatalperiode. Zntralbl Gynaekol, Leipzig, 98 (26): 1618-25, 1976.

LISBOA, A. M. J. Cuidados imediatos ao recém-nascido. In: EMERGENCIAS no perfodo neonatal. São Paulo, Centro de Estudos Perinatais de São Paulo, 1968.

MARAZZINI, P. \& SANTUCCI, S. Indice di Apgar, morbosità, mortalità neonatale rilevanti del periodo 1970.5 presso l'Ospedale di Matenta. Minerva Pediat, Torino 29 (27): 1683-8, 1977.

MARX, G. F. et alii. Correlation of biochemical data with Apgar Scores at birth and one minute. Brit J. Anaesth, Cheshire, 49 (8): 831-3, 1977.

MAYRHOFER, D. Die Reanimation des asphyktischen Neugeborenen. Der Anaesthesist, Berlin, 20: 253-5, Jul 1971 .

NAYE, R. L. Underlying disorders for the neonatal deaths associated with low Apgar Scores. Biol. Neonat, Basel, 35 (3-4): 150-5, 1979.

SERUNIAN, S. A. \& BROMAN, S. H. Relationship of the Apgar Scores and Bayley mental and motor scores. Child Development, Lafayette, 46 (3): 696-700, 1975.

YEH, S. et alii. A study of the relationship between Goodwin's high-risk score and feta loutcome. Am J Obstet Gynecol, St. Louis, 127 (1): 50-5, 1977.

ZIEGEL, E. \& CRANLEY, M. Assistencia clínica durante o parto. In Rio de Janeiro, Interamericana, 1980. Parte 15, p. 285-7. Enfermagem obstétrica, 